\title{
Zur Lehre von der Graviditas interstitialis.
}

\author{
Von
}

\author{
G. Leopold, \\ Privatdocent in I.nimim \\ (Mit Abbildung $\mathrm{T}_{\text {w1w. }} \ldots$,
}

Mit den folgenden Zeilen füge ich den wenigen bekannten Fällen von Schwangerschaft in der Gebärmuttersubstanz eine neue Beobachtung hinzu, welche ganz besonders durch den klinischen Verlauf, sowie durch ihre anatomischen Befunde von den bisherigen Fällen erheblich abweicht. Zeichnet sich diese Beobachtung schon dadurch aus, dass sie die Ursache der Schwangerschaftsanomalie ziemlich klar darlegt, so erregt sie andererseits durch einzelne Symptome, wie sie bei anderen Fällen von Extrauterinschwangerschaft meines Wissens nicht zur Geltung kamen, das diagnostische Interesse.

Frau D., 36 Jahre alt, hat drei Mal geboren, zuletzt vor einem Jahre. Vor drei Jahren erlitt sie einen schweren Abort im dritten Monate mit so starken Blutungen, dass sie sich nur langsam davon erholte. Am 24. Juni 1878 trat nach einer Pause von zehn Wochen eine leichte Blutnng auf, welche von der Familie der Frau als beginnender Abort gedeutet wurde. Doch glaubte Frau D. die Möglichkeit einer Schwangerschaft aus dem Grunde mit aller Gewissheit aussehliessen zu können, weil sie diesmal, entgegen den sofort post conceptionem anfgetretenen, erheblichen subjectiven Beschwerden in den früberen Fällen, nicht die geringsten Andeutungen davon hatte.

Am 26. Juni nun wurde sie über Mittag nach einer körperlichen Anstrengung plötzlich von einer so profusen Gebärmutterblutung befallen, dass sie in kurzer Zeit ziemlich ein halbes Waschbecken Blut verloren haben mochte. Der herbeigerufene College, Herr Dr. Sänger, hatte wegen der drohenden Erschöpfung und bedeutenden Anämie nur so viel Zeit, um eiligst die Scheide völlig 
zu tamponiren. In den Blutmassen fand ich mit ihm bei eingehendster Durchsuchung weder Eihäute noch Stücke derselben, noch einen Fötus, sodass nach diesem negativen Befunde der Blutung, welche uns, und gewiss jeden Anderen, nach der Anamnese zunächst an eine Abortblutung denken liess, selbstverständlich ein noch im Uterus befindliches Ei zu erwarten war.

Aber schon die äussere Untersuchung der Frau nach der Tamponade ergab einen interessanten Befund, welcher, zunächst ohne bestimmte Begründung, mir eine uterine Schwangerschaft zweifelhaft machte. Rechts vom Fundus uteri nämlich, welcher ungefähr in Nabelhöhe stand, füblte man deutlich durch die schlaffen Bauchdecken einen circa hühnereigrossen, breitbasigen, wenig empfindlichen Tumor, wie man ihn ab und zu bei Gebärenden und Wöchnerinnen beobachtet, und welcher sofort als breitbasiges, subseröses Fibrom imponirte. Da die sehr intelligente und gut beobachtende Patientin so bestimmt die Unwahrscheinlichkeit einer Schwangerschaft hinstellte, so regte jener fibromähnliche Tumor wiederholt in mir die Frage an, ob nicht eine so beträchtliche Blutung auch mit einem Fibrom, neben welchem sich vielleicht noch ein gleich grosses submucöses vorfinde, im Zusammenhange stehen könne.

Dieser Zweifel an einer Schwangerschaft erhielt nun durch den Weiterverlauf seine Begründung. Denn als am 27. die Tampons entfernt waren, hatte sich der Uterus wesentlich verkleinert. Puls, Temperatur und Respiration waren normal. Vòn äusserer Blutung war kaum Etwas bemerkbar und die Kräfte fingen an sich zu heben. Bei der inneren Untersuchung gelangte man leicht durch das weit geöffnete Collum ein Stück bis über den inneren Muttermund, über welchem in der Uterushöhle von einem Eie Nichts zu finden war. Da aber ein stärkerer Druck bei der bimanuellen Untersuchung auf den Fundus uteri, mithin auf jene Geschwulst absichtlich vermieden wurde, so blieb vorläufig noch unentschieden, ob nicht wenigstens kleine Reste von dem angenommenen Eie im Fundus geblieben waren.

Am folgenden Tage sistirte die Blutung völlig. Der Uterus und jene Geschwulst hatten sich noch mehr verkleinert; der Fundus stand drei Finger breit unter dem Nabel. Bei dieser Verkleinerung des Uterus ist jedoch nicht zu vergessen, dass von nun an keine Tamponaden mehr stattfanden, der auffällige Hochstand der Gebärmutter bei der ersten Untersuchung daher von der Tamponade herrüluren konnte.

Nachdem sich Patientin nun vom 29. Juni bis 3. Juli bei absoluter Bettruhe fieberlos and im Allgemeinen befriedigend befunden, auch etwas erholt hatte, trat am 4. Juli Nachmittags eine nene Blutung ein. Da der Hausarzt der Familie an der Annahme eines normalen Abortes noch festhielt und den Grund der neuen Blutung in Placentarresten sah, so legte er, um den inzwischen wieder geschlossenen Cervicalkanal zu erweitern, am Abend des 4. Juli den Colpeurynter ein. Darauf sei in der folgenden Nacht eine placentaähnliche Masse 
abgegangen, welche leider nicht aufgehoben wurde. Am 5. und 6. Juli habe die Blutung in Gleichem noch mässig fortgedauert; in Folge dessen sei der Colpeurynter noch liegen gelassen worden.

Als ich am 7. Juli die Kranke wieder sah, hatte sie eben einen tüchtigen Frost gehabt; 40,0 Temperatur, 120 Puls. Geröthete Wangen ; mässig empfindlicher Leib; brennender Durst; grosse Unruhe; im Uebrigen die Zeichen einer beginnenden septischen Peritonitis. Da jetzt erst recht zurückgebliebene und eventuell verjauchende Placentarreste als Ursachen der Entzündung beschuldigt wurden, so überzeugte ich mich nochmals durch eine vorsichtige, vollständige Austastung der Gebärmutterhöhle, dass keine Spur eines Fremdkörpers, vor All em aber keine Spur einer Placentarstelle aufzufinden war. Bemerkenswerth blieb nur bei der Untersuchung, dass der Zeigefinger in der an und für sich schon weiten Uterushöhle nach rechts oben, entsprechend der äusseren Geschwulst, eine starke Ausbuchtung fand.

Fortwährende Eisumschläge, starke Dosen Chinin und Wein, Carbolausspülungen änderten Nichts in dem Weiterverlaufe der Peritonitis. Am 8. Juli erhielt sich die Temperatur unter mehrmaligen Frösten zwischen 40 und 41 Grad, Puls 120-130. Am Morgen des 9. Juli trat unter den Zeichen einer bedenklichen Anämie ein Collaps ein, Temperatur 37,6, Abends 39,0. Puls 140. Dabei der Leib über und besonders rechts vom Uterus sehr schmerzhaft. Sensorium unbenommen; aussergewöhnlich geistige Regsamkeit. Die Kranke fühlt bestimmt die Nähe ihres Todes und stirbt ruhig am Morgen des 10. Juli.

Bei der am Nachmittage stattfindenden Section(Herr Dr. Hu ber) erwartete ich nach dem klinischen Verlaufe einen vergrösserten Uterus mit wunder Schleimhaut, aber ohne Placentarstelle; rechts am Fundus uteri einen fibromähnlichen, entzündeten Körper und die Zeichen einer frischen Peritonitis.

Nach der Eröffnung der Bauchhöhle bedeckten zunächst frisch entzündlich verklebte Darmschlingen die Beckenhöhle. Sie wurden nach aufwärts gezogen. Dabei entwickelten sich besonders zwischen Colon ascendens und transversum dicke Schichten alter und frischer, geronnener Blutmassen, welche sich nach der rechten Uterusgegend fortsetzten und von hier aus ihren Ursprung nahmen. Doch betrugen diese Blutergüsse nur ein mässiges Quantum und erreichten kaum die Hälfte von den Blutmassen, wie man sie für gewöhnlich bei Tubenschwangerschaft mit Berstung des Fruchtsackes zu sehen bekommt.

Nach Freilegung der Beckenhöhle präsentirte sich nun sehr schön der hypertrophische Uterus mit einer rechts am Fundus befindlichen, hühnereigrossen, aufgebrochenen Geschwulst. Die Oberfläche derselben war höckerig und sah so eigenthümlich aus, dass Herr 
Dr. Huber mit Recht das ganz treffende Bild einer angeschnittenen, frisch gekochten Blutwurst dafür brauchen konnte. Nach Herausnahme des ganzen Genitaltractus zeigte sich nun (s. Abbildung), dass der Uterus $11 \mathrm{Cm}$. lang war und sich rechts oben in eine aufgebrochene Masse fortsetzte, auf welche die Serosa uteri bis zum mittleren Umfange sich deutlich fortsetzte. Hier endete die Serosa in einer zackigen, vielfach eingerissenen Linie; sie erschien auseinandergeborsten durch eine zunächst verworrene, blutdurchtränkte und zerfetzte Masse. Ein Längsschnitt durch Uterus und Geschwulst ergab nun, dass die Gebärmutterkörperhöhle 5, die der Cervix $3^{3 / 4} \mathrm{Cm}$. lang war. Letztere war ausgekleidet von einer durchaus intacten Schleimhat, welche am inneren Muttermunde scharf absetzte. Von hier an erstreckten sich nun die flottirenden und lebhaft injicirten Deciduafetzen durch die ganze Gebärmutterhöhle. Diese letztere stand in keinem Zusammenhange mit der aufsitzenden Geschwulst; insbesondere war von einer Einmündung oder von einem Uebergange starker Blutgefässe nicht das Geringste bemerkbar.

Die durchschnittene Geschwulst lässt sich am besten mit einer Halbmondform vergleichen, dessen Spitzen der geborstene Pol darstellt. In der breiten Basis klaffen zahlreiche kleinere und grössere Blutgefässe; die letzteren sind fast sämmtlich ausgestopft mit Convoluten ron Chorionzotten, welche ziemlich frei in ihnen flottiren und nur mit einzelnen Köpfen in der Gefässwandung festhaften. Somit haben wir es hier mit einer Placenta zu thun, welche aus kleinsten, zwischen den Muskelbündeln des Uterus eingenisteten Inseln besteht, die sich ein Stück aufwärts nach den Halbmondhörnern fortsetzen. Ist nun schon diese Placenta von Blutergüssen stark durchsetzt, so sind es noch mehr die oberen Wände der Geschwulst, in deren äusseren Schichten man deutlich die von der Gebärmuttersubstanz heranziehenden Muskelbündel, in deren inneren Schichten man leicht dicke Blutergüsse erkennt. In Folge dessen ist die von einem Chorion ausgekleidete Geschwulsthöhle von allen Seiten zusammengepresst, und gleicht genau einer uterinen Eihöhle, bei welcher nach dem frühen Untergange des Fötus Blutergüsse in Placenta und Eihäute erfolgt sind.

Am oberen Eipole sind nun die feinen Wandungen, wie es scheint, schichtweise aufgebrochen; doch ist bemerkenswerth, dass die Eihöhle selbst nicht geöffnet ist. 
Beide Tuben sind normal gebildet und für eine feine Sonde bis zur Uterusmuskulatur resp. bis zum Fruchtsack leicht durchgängig. Die Ovarien sind gross und derb; im rechten befand sich nach hinten, am uterinen Ende ein ungefähr kirschengrosses Corpus luteum mit etwas gefalteter, gelblicher Rinde.

Unterlag es somit keinem Zweifel, dass wir es hier mit einer interstitiellen Schwangerschaft zu thun hatten, so war zunächst von grösstem Interesse die Frage nach den Ursachen dieser seltenen Anomalie. $\mathrm{Da}$ die sonst wohlgebildeten Genitalien weder durch Adhäsionen, noch durch Verwachsungen oder Abknickungen an Tubien oder Ovarien irgend etwas Abnormes zeigten, so liess sich vorerst eine Anomalie im Inneren jener Organe erwarten. Daher wurden die Tuben, da die Durchforschung ihres Lumens mit einer feinen Borste selbstverständlich keinen genügenden Aufschluss geben kann, mit einem scharfen Rasirmesser, besonders von der Einmündung in den Uterus an, Schnitt auf Schnitt verfolgt. Hierbei zeigte sich folgendes:

Was zunächst die linke Tube anlangt, so läuft ihr Lumen, nachdem sie die Seite des Uterus erreicht hat, zunächst ungefähr einen Centimeter weit über den Fundus uteri, ziemlich dicht unter der Serosa hin, und knickt sich dann mit einem Male im rechten Winkel so ab, dass ihr Lumen innerhalb der Muskulatur des Fundus uteri nunmehr gerade nach abwärts läuft und dabei sich von $1 \mathrm{Mm}$. Durchmesser auf circa $3 \mathrm{Mm}$. erweitert. Somit öfnet sich das Uterinende der Tube, umlagert von einzelnen Deciduafalten, vom linken oberen Winkel der Gebärmutterhöhle, dem gewöhnlichen Eintrittsorte, mindestens $1 \mathrm{Cm}$. entfernt, und somit weicht der Tubenkanal von der gewöhnlichen Durchgangsrichtung hier erheblich ab.

Auf der rechten Seite macht die Verfolgung des Tubenkanales viel mehr Schwierigkeiten. Hier lässt sich nur durch eine sorgfältige schnittweise Durchmusterung Klarheit erzielen. Nachdem der Tubenkanal in die Muskulatur des Uterus eingetreten ist, zieht er sich in stark convexen Bogen hinten auf dem Fruchtsacke dicht unter der Serosa bis zur halben Höhe der Eihöhle hin, und biegt dann ebenfalls scharf, im rechten Winkel nach unten um, indem er sich aber hierbei beträchtlich erweitert, auf ungefähr $1 \mathrm{Cm}$. Länge mit 4-5 länglichen Schleimhautpolypen a usgestopft ist und sofort in die weite, von Blutmas- 
sen ausgefüllte Eihöhle übergeht. Hier verliert er sich sofort und ist von einer Fortsetzung des Kanals in die Gebärmutterhöhle Nichts aufzufinden.

Bei einer wiederholten Betrachtung dieser Verhältnisse, und namentlich wenn man die völlige Abtrennung der Eihöhle von der Uterinhöhle berücksichtigt, kommt man immer mehr zu der Annahme, dass jedenfalls durch mechanische Behinderung, und zwar durch die dicht aneinander gepressten Polypchen, das Ei in dem Endstücke des Kanales sitzen geblieben ist und sich hier festgenistet hat.

Rechterseits läuft das Ligamentum rotundum nach'aussen vom Fruchtsack' nach abwärts. Aus den Mammae konnte, wie auch an der Lebenden, Secret nicht ausgedruickt werden.

Betreffs der mikroskopischen Verhältnisse in den Tuben, in der Decidua vera, wie in der Schleimhaut des Cervicalkanales kann ich auf das in meinen früheren Arbeiten hierüber Erwähnte verweisen.

Von besonders grossem Interesse ist die Thatsache, wie ich sie schon in einem früheren Falle von Tubenschwangerschaft constatirt habe ${ }^{1}$ ), dass die Köpfe der grösseren Chorionzotten, oder die Haftwurzeln derselben anstatt in die Decidua serotina, sich zwischen die Muskelbündel eingegraben haben. Dem entspricht auch die anderweite, höchst interessante Beobachtung, dass in die erweiterten Uterinvenen die Chorionzotten eingebrochen und nur von ihrem eigenen Epithel bedeckt sind. Somit haben sie offenbar, gerade so wie ich es neuerdings für die Venen der Decidua und die grossen Bluträume der Placenta dargestellt habe ${ }^{2}$ ), das Endothel der oberflächlichen Capillaren und Uterinvenen auseinandergedrängt und sind eingewuchert, ohne von einem mütterlichen Epithel noch bekleidet zu sein. Daher geschieht auch bier, wie bei normaler Schwangerschaft die Trennung des fötalen und mütterlichen Blutes nur durch das Stroma und Epithel der Zotten selbst.

Fassen wir nun das Ergebniss der Section zusammen, so handelte es sich hier u m eine rechtsseitige interstitielle Schwangerschaft mit einem geborstenen Fruchtsacke von der Grösse des zweiten Monates, mit fehlendem Fötus und Amnion; zweitens um alte und frische Blut-

1) Dieses Archiv, Bd, X, S. 248.

2) Dieses Archiv, Bd. XI, S. 443. 
ergüsse in der Umgebung der Gebärmutter, und drittens um eine frische Peritonitis.

Die Epikrise dieses Falles erfordert zunächst einige Worte über die Aetiologie der vorliegenden Schwangerschaftsanomalie. „Die Ursachen der tubaren und peritonealen Schwangerschaft sagt Spiegelberg (Lehrbuch der Geburtshülfe, 1878, S. 308), können nur in Hindernissen, welche eine Leitung des Eies in und durch die Tube hindurch unmöglich machen oder erschweren, liegen, oder sie sind in der sogenannten Ueberwanderung des Eies zu suchen. Für alle sonst etwa angenommenen Ursachen (dynamische) lässt sich auch nicht der Schatten eines Beweises beibringen; und selbst die erwähnten Hindernisse nachzuweisen, gelingt bei Autopsien nur in einzelnen Fällen, weil eben durch die extrauterine Entwickelung an den inneren Genitalien die eingreifendsten Veränderungen erzengt und primäre Anomalien verwischt werden. Die Obstruction der Tube kann eine perfecte sein oder nur in einer Verengerung bestehen;“ - , ,sie ist meist durch peritoneale Adhäsionen und Bänder, welche die Tube unnatïrlich fixiren und abknicken, bedingt; es entspricht dem der Umstand, dass die Extrauterinschwangerschaft in den meisten Fällen Mehrgeschwängerte betrifft und dass diese Jahre vorher relativ steril waren; jene Anomalien datiren eben meist aus früheren Puerperien, erschweren die Conception; es entspricht ihm ferner die ron Hecker hervorgehobene Thatsache von dem überwiegend linksseitigen Sitze der Tubenschwangerschaft - linksseitige Beckenentzündungen sind bekanntlich häufiger, als rechtsseitige. In einzelnen Fällen lag das Hinderniss für die Eileitung in Polypen, welche die uterine Mündung des Eileiters verlegten, wie Breslau und Beck beobachteten, oder in intraparietalen Fibroiden des Uterus (Magrath, Roth). Relativ häufig auch sind Zwillinge bei Eileiterschwangerschaft gesehen, und es ist nicht unmöglich, dass die beiden Eier sich gegenseistig auf ihrer Wanderung aufhielten."

Unser Fall passt nun, von einzelnen Modificationen abgesehen, in den Rahmen dieser Schilderungen sehr gut hinein. Denn es handelt sich hier um eine Mehrgebärende, die ziemlich. schnell nacheinander, zuletzt vor einem Jahre geboren hat und welche in den Beckenorganen, speciell den Tuben nicht die geringsten 
Spuren alter Entzündungen oder Adhäsionen aufzuweisen hatte. Somit hat, man könnte sagen die Ausnahme von der Regel, dass hier der extrauterine Fruchtsack auf der rechten Seite lag, etwas viel mehr Befremdendes, und unwillkürlich sucht man den Grund des abnormen Eisitzes in mechanischen Hindernissen.

Diese letzteren können sich nun meines Erachtens kaum schöner und unzweideutiger auffinden lassen, als in jenen kleinen Polypen, welche das Uterinende der rechten Tube ausstopfen und bis in die Eihöhle deutlich zu verfolgen sind. Der Vorgang der Eibehinderung wịd dann der gewesen sein, dass das befruchtete Eichen vor den an einander gepressten Polypen liegen blieb und sich nun in der etwas wuchernden Tubenschleimhant einbettete. Das wachsende Ei drängte dann die Schleimhautfältchen auseinander, wucherte in die Venen der Uterusmuskulatur ein, um sich eine Placenta zu bilden, und trieb allmälig die Muskelbündel wie die Serosa immer mehr auseinander, bis eine Berstung erfolgte.

Man wird vielleicht hier einwerfen wollen, dass jene Polypen nur Producte der Schwangerschaftswucherung seien. Doch ist dieses vermeintliche post hoc propter hoc hier, wo die Verhältnisse so klar liegen, entschieden zurückzuweisen; es würde wenigstens sehr auffällig bleiben, warum denn gerade im Uterinende der rechten Tube und nicht auch der linken solche Schleimhautwucherungen entstanden sind. Ein zweiter Einwurf, warum denn nicht schon bei früheren Schwangerschaften ein abnormer Sitz des Eies eintrat, lässt sich leicht damit entkräften, dass die Polypen erst nach der letzten Entbindung entstanden sein können, oder das zweitens das $\mathrm{Ei}$ in früheren Fällen die linke Tube passirt haben kann.

Somit mehren sich mit diesem Nachweise die Fälle, in denen kleine Polypen die Ursache von Tuben-, resp. interstitieller Schwangerschaft waren. Besonders bekannt ist in dieser Hinsicht der Fall von Beck; ferner der von Breslau. Es möge hierbei nicht unerwähnt bleiben, dass auch in dem früher von mir beschriebenen Falle von Graviditas tubo-abdominalis (1. c.) in den Uterinenden beider Tuben sich je ein, resp. zwei linsengrosse Polypen vorfanden, welche, wie hier, den Eintritt des befruchteten Eichens hätten verhindern können.

Was nun den klinischen Verlauf and die Symptome in der zu Grunde liegenden Beobachtung anlangt, so ist zunächst 
zu bemerken, dass die Frau, welche in früheren Schwangerschaften durch eine Menge subjectiver Beschwerden ganz bestimmt den Eintritt der Schwangerschaft angeben konnte, diesmal keine einzige beobachtet hatte, daher auch die Wahrscheinlichkeit der Gravidität nicht zugab. Dieser Umstand exklärt sich selbstredend nicht durch den extrauterinen Sitz des Eies, sondern jedenfalls durch das Fehlen des Fötus, welcher am Ende des ersten, vielleicht am Anfange des zweiten Monates abgestorben und dann resorbirt worden ist. Sehen wir doch in Fällen von normaler Schwangerschaft die ganz gleichen Erscheinungen, dass mit dem Tode der Frucht in den frühen Schwangerschaftsmonaten jede subjective Beschwerde nicht selten vollständig verschwindet.

Somit blieb die Eihöhle in ihrer Entwickelung stehen. Die Chorionzotten hingegen wucherten weiter und bildeten eine Art feinster Placentarinselchen, welche zusammengenommen einer Placenta aus dem dritten Monate entsprechen würden. Im Weiterverlauf kam es, wie so oft nach dem Untergange des Fötus, zu Blutergüissen in die Wände des Fruchtsackes, welche eine immer stärkere Spannung der Serosa herbeiführten.

Als nun am 24. Juni nach einer körperlichen Anstrengung eine leichte uterine Blutung erfolgte, so trat damit eine Lockerung der Decidua vera, aber gleichzeitig ein oberflächlicher Riss in der Serosa des Fruchtsackes ein, aus dem sich eine geringe Menge Blut in die Bauchhöhle ergoss. Es fand sich daselbst an den tiefsten Stellen als alte, geronnene Masse.

Nachdem zwei Tage hierauf eine neue Anstrengung erfolgt war, trat sofort danach eine sehr schwere äussere Blutung ein. Wenn diese schon genügte, um die beträchtliche Anämie der Kranken zu erklären, so wies erstens kein einziges Symptom auf eine eventuelle stärkere Bauchhöhlenblutung hin, zweitens liess sich auch nach dem Sectionsbefunde keine solche annehmen. Wie erklärt sich nun dieses geradezu frappante, umgekehrte Verhältniss, der äusseren schweren Blutung bei Tubenschwangerschaft? Da, wie schon oben erwähnt, keine einzige Communication zwischen Uterin - und Fruchthöhle, auch kein directer Gefässübergang besteht, so bleibt nur die eine Annahme übrig; dass sich plötzlich, wie bei einem normalen Aborte, die Decidua vera in toto in ihrer Drüsenschicht lockerte, und somit eine grosse Flächenblutung erfolgte, welche durch die benachbarten Placentaruterinvenen reichliche Zufuhr erlangt haben wird. Es wird 
daher die Decidualmasse in den ausgestossenen Blutmengen zertrümmert gelegen haben. Ein allmäliger, stossweisser Abgang von Decidua wurde niemals beobachtet.

Soweit ich die Literatur der Tuben-, speciell der interstitiellen Schwangerschaft durchsehen konnte, habe ich nirgends eine Notiz von einer so profusen Uterinblutung, welche ganz hauptsächlich die Diagnose irre leitete, gefunden. Hennigl) erwähnt nur, dass eine untypische Blutung aus der Scheide die Tubenschwangerschaft hin und wieder begleitet. „In drei Fällen bestand neben der inneren zugleich eine äussere Metrorrhagie; in elf Fällen war die Blutung lediglich eine äussere." Doch ist von der Schwere der äusseren Blutung und der dabei befindlichen inneren geringen Blutung nichts erwähnt.

Nachdem sich nun die Kranke nach der Tamponade von dem Blutverluste etwas erholt und einige Tage sich wohl befunden hatte, mittlerweile auch der durch die Bauchdecken fühlbare Fruchtsack verkleinert war, traten von Neuem Blutungen ein, welche wiederholte Tamponaden der Scheide und Austastungen der Uterinhöhle veranlassten und hiermit meines Erachtens das Ende der Kranken beschleunigten. Denn bald nach oder durch die Eingriffe traten die lebhaftesten Zeichen einer septischen Peritonitis und einer bedenklichen Bauchhöhlenblutung auf, welcher die Kranke schnell erlag. Und in der That konnten die ganz frischen Blutergüsse, welche aus den stärker aufgebrochenen Eiwänden stammten, ebenso wie die peritonitischen Auflagerungen nur wenige Tage alt sein.

Betreffs der Diagnose dieses lehrreichen Falles drängen sich zwei Fragen auf. Erstens, was hat in der Diagnose irre geleitet? und zweitens, was hätte trotz der schwierigen Verhältnisse zur richtigen Diagnose führen können?

Irre geführt hat hauptsächlich die beträchtliche äussere Blutung, ferner die anfänglich fehlende Bauchhöhlenblutung, drittens der mangelnde sichtbare Abgang von Deciduafetzen, viertens der für ein Fibroid gehaltene Tumor ${ }^{2}$ ) und besonders die mit dem Aufhören der Blutung gang auffällige Rückbildung desselben, und endlich fünftens die für die Zeit der Schwangerschaft auffallende Kleinheit des Fruchtsackes.

1) Die Krankheiten der Eileiter und die Tubenschwangerschaft. Stuttgart 1876, Enke. S. 123.

2) cf. Hennig, l. c., S. 148. 
Diesen Momenten gegenüber jedoch hätten nach dem Sectionsergebnisse und klinischen Verlaufe folgende Zeichen zur Diagnose führen können: Erstens das Ausbleiben der Menses, ferner der hypertrophische Uterus mit seiner weichen und offenen Cervix, drittens das Fehlen der Placentarstelle im Uterus, und viertens die vor dem Tode auftretende innere Blutung.

Hätte sich mit Hülfe dieser Symptome die richtige Diagnose stellen lassen, so wäre gerade in diesem Falle, wo der Sitz, die Grösse and die übrigen anatomischen Verhältnisse des Eisackes aussergewöhnlich günstige sind, in therapeutischer Hinsicht ein chirurgischer Eingriff des Versuches unbedingt gelohnt. In Anbetracht des fast sicher letalen Ausganges der interstitiellen Schwangerschaft hätte hier nach Eröffnung der Bauchhöhle der Eisack mittels eines Zirkelschnittes durch die Muscularis uteri aus dieser herausgehoben werden müssen. Danach hätte man die Uteruswunde durch starke Seidennähte zu schliessen gehabt.

Es unterliegt wohl keinem Zweifel, dass bei der vollendeten jetzigen Technik der Chirurgie ein derartiger Versuch gerechtfertigt ist und in einzelnen günstigen Fällen Aussicht auf Erfolg verspricht. 


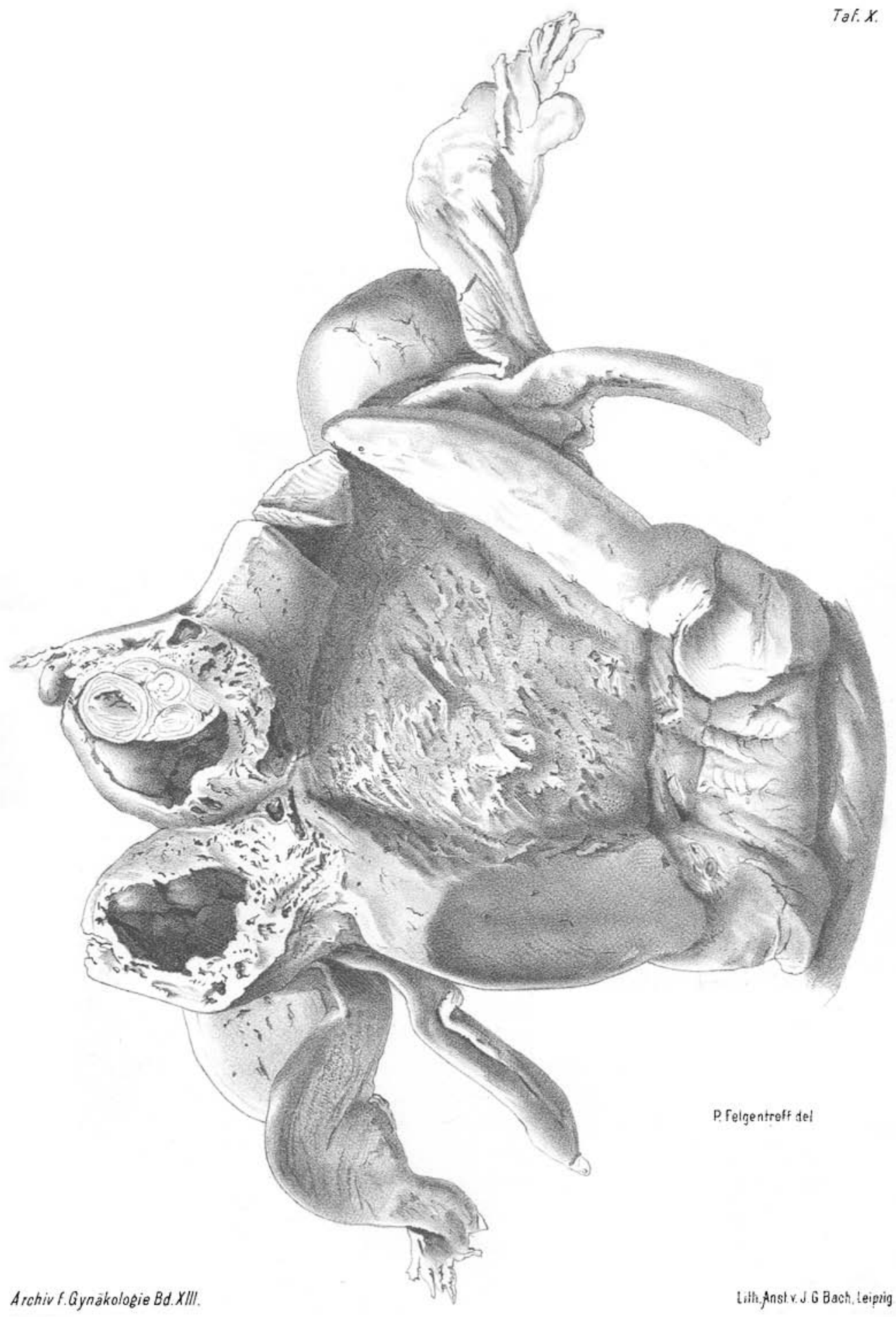

